\title{
DYNAMO DRIVEN ACCRETION DISCS AND DWARF NOVA ERUPTIONS
}

\author{
P. J. ARMITAGE ${ }^{1}$, M. LIVIO ${ }^{2}$, J. E. PRINGLE ${ }^{1,2}$ \\ 1. Institute of Astronomy, Madingley Road, Cambridge, \\ CB3 OHA, UK \\ 2. Space Telescope Science Institute, 3700 San Martin \\ Drive, Baltimore, MD 21218, USA
}

\section{Introduction}

A magnetic dynamo whose crucial ingredient is the Balbus-Hawley instability (Balbus \& Hawley 1991) is a promising candidate for generating accretion disc viscosity. Both semi-quantitative analyses (Tout \& Pringle 1992) and simulations (e.g. Brandenberg et al. 1995) suggest that such dynamos can operate in a disc environment. Here we aim to apply simple dynamo ideas to models of dwarf novae, and in particular investigate how dynamo driven discs can produce different values for $\alpha$ in quiescence and in outburst.

\section{Generating outbursts}

The Balbus-Hawley instability operates with an arbitrarily weak vertical field, so a dynamo requires no pre-existing strong fields or disc turbulence. However a strong field,

$$
B_{\mathrm{Z}}^{2}>\frac{24}{\pi} \rho c_{\mathrm{S}}^{2}
$$

where $B_{\mathrm{Z}}$ is the vertical field, $\rho$ the density, and $c_{\mathrm{S}}$ the sound speed shuts off the instability. Therefore rapid cooling at the end of a dwarf nova outburst may act to depress the thermal energy relative to the magnetic energy, shut off the instability, and quench dynamo action. The turbulence generated by the dynamo in the disc could then die away, leaving a quiescent disc with a relatively strong vertical field and very low viscosity. The dynamo could then be restarted (and a new outburst begin), when sufficient mass had been added at the outer edge to violate inequality (1). 

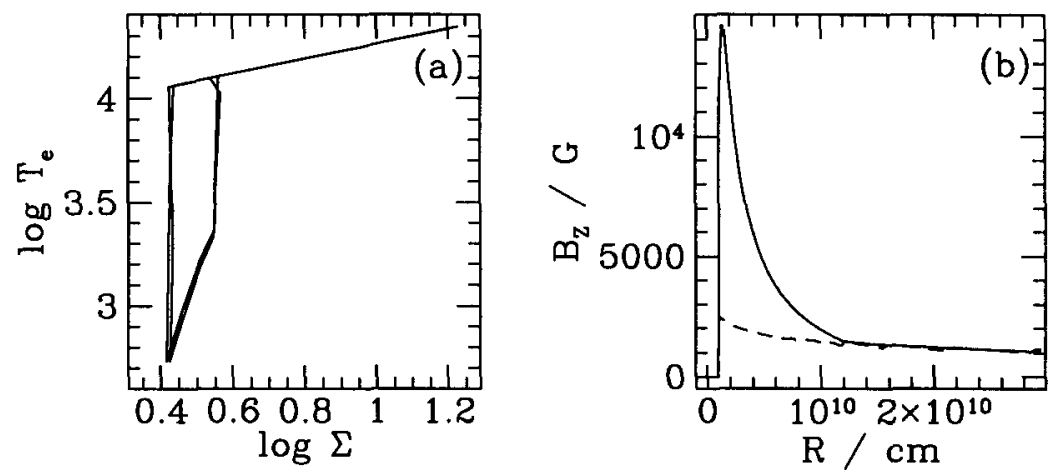

Figure 1. (a) Path traced out in the effective temperature-surface density plane by an annulus at the inner edge of the disc. (b) Vertical magnetic field strengths during outburst (solid line) and quiescence (dashed line).

\section{Results}

We have modified a time-dependent disc code similar to that described by Cannizzo (1993) to model outburst cycles generated by the above mechanism. The details of the code are described elsewhere (Armitage, Livio \& Pringle 1996). We can produce well-separated outbursts provided that the working dynamo generates an $\alpha$ of $\sim 0.2 \ldots 0.3$. Our model differs from the normal thermal instability in not requiring a double valued ' $\mathrm{S}$-curve' in the surface density-temperature plane, as the transition to the hot state is controlled by a magnetic trigger condition [inequality (1)]. Fig. 1a shows the path followed by an annulus in our model, with a prolonged cooling phase at constant surface density in quiescence. Observational consequences include:

- An active disc corona, as dissipation is concentrated in low density regions of the disc (leading also to a different vertical thermal structure).

- Magnetic fields (on scales of order the disc scale height) of a few kG in quiescence and reaching up to $\sim 10 \mathrm{kG}$ in outburst [see Fig. 1b].

- Flickering due to variability in dynamo 'cells' across the disc.

- A cold quiescent disc, with a surface density profile strongly weighted towards the outer edge as the next outburst approaches. The temperature profile in the quiescent disc is flat.

\section{References}

Armitage, P.J., Livio, M., Pringle J.E., 1996, Ap. J., in press

Balbus, S.A., Hawley, J.F., 1991, Ap. J., 376, 214

Brandenburg, A., Nordlund A., Stein, R.F., Torkelsson U., 1995, Ap. J., 446, 741

Cannizzo, J.K., 1993, Ap. J., 419, 318

Tout, C.A., Pringle J.E., 1992, MNRAS, 259, 604 